\title{
Bypassing bleaching with fluxional fluorophores
}

\author{
Combining photoactivation and spontaneous blinking enables long time-lapse localization microscopy.
}

S ingle-molecule localization microscopy (SMLM) offers the highest resolution among the standard super-resolution microscopy approaches. In SMLM, final images are built up from hundreds to thousands of image frames in which only a small fraction of the total fluorophores are captured. Light is typically used to randomly switch on a small subset of the fluorophores per imaging round, making localization microscopy light intensive. These light doses and long acquisition times can lead to phototoxicity, which limits the applicability of these methods for live-cell imaging. Long time-lapse live-cell imaging with SMLM using conventional fluorophores is further limited because of photobleaching.

Pablo Rivera-Fuentes and graduate student Elias Halabi at ETH Zurich sought to develop a labeling strategy that would enable extended time-lapse super-resolution imaging by limiting photobleaching and phototoxicity. They also wanted their approach to be compatible with diverse cellular environments, such as low $\mathrm{pH}$. To reach this goal, they combined two ideas that have previously been explored in SMLM: photoswitching and spontaneous blinking.

For this, they used rhodamine $\mathrm{B}$, a dye that is fluxional. Practically speaking, this means that the dye exists in thermal equilibrium between on and off states and can spontaneously switch between them. They engineered the dye to be photoswitchable by attaching an acylhydrazone unit. Prior to light exposure, the dye is dark and does not blink. However, upon exposure to 410 -nm light, the dye is turned on and able to blink at random. This photocontrol is crucial to the success of the approach, as the light dose used for photoswitching precisely determines the amount of total fluorophore that is able to blink-a parameter that is closely linked to both imaging speed and resolution. Additional rounds of photoswitching can also introduce 'fresh' dye for imaging, bypassing the photobleaching of the previously imaged dyes.

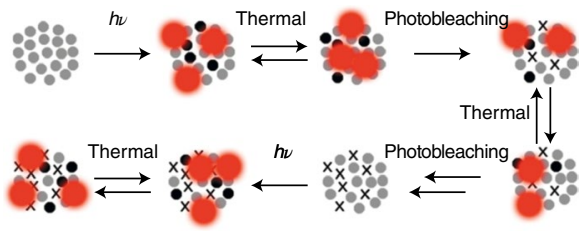

Schematic depicting the principle behind the combination of photoswitching and spontaneous blinking used for SMLM. Adapted with permission from Halabi et al. (2019), Springer Nature.

Once the idea of combining photoswitching and fluxionality was in hand, "the greatest challenge was to come up with a fluorophore that could do that," notes Rivera-Fuentes. The ideal dye needed to be completely off before photoswitching, but then blink brightly and rapidly after light exposure. "It took us several rounds of molecular design, synthesis, and evaluation before we found the combination of rhodamine and acylhydrazone that eventually behaves in the desired way," he recalls.

To validate that the photoswitching dye, called PFF-1, worked as they expected, they imaged it in the context of polymer films, and saw that PFF-1, but not rhodamine $\mathrm{B}$, exhibited blinking after initial light exposure. They then tested the dye in cells, where they wanted to study the biology of acidic compartments. These are challenging to study with existing dyes known to blink in hydrophobic environments. Another dye, hydroxymethyl silicon rhodamine (HMSiR), has been used in previous long time-lapse studies of subcellular compartments. However, "HMSiR is incompatible with low-pH environments such as those found in lysosomes or synaptic vesicles," Rivera-Fuentes says. "Under these conditions, too many molecules are found in the fluorescent form; their signals overlap and cannot be resolved."

For their experiments, they targeted PFF-1 to lysosomes in HeLa cells and carried out both 2D and 3D SMLM. They could track lysosomes with good spatiotemporal resolution over thirty minutes, even with only a single pulse of light at the beginning of the experiment, but found that treatment with light every ten minutes led to reduced photobleaching and more signal over time. They also showed single-vesicle tracking in human neurons with sub-second time resolution. Their findings revealed that different vesicles display a range of diffusion coefficients, and that they can move along tracks and accumulate in hot spots, as previously observed. Their imaging method also allowed them to observe, for the first time, that vesicles can hop between hot spots, which seem to be connected by tracks along which the vesicles move. This could allow neurons to have a constant pool of localized vesicles, while still allowing rapid exchange.

Although the demonstrations shown highlight the potential impact of the development, Rivera-Fuentes points out several important future directions. Beyond showing that the approach works generally beyond acidic compartments, his team is interested in showing that the method is more broadly useful for labeling specific targets, such as proteins of interest. For this, he envisions using Halo- or SNAP-tag technologies and "identifying molecules that become fluxional only when they are bound to their target, which would simplify the labeling procedure and minimize non specific background." He also hopes to develop similarly performing dyes in other colors. Taken together, these advances would be a major step forward for live-cell super-resolution imaging.

\section{Rita Strack}

Published online: 30 April 2019

https://doi.org/10.1038/s41592-019-0402-2

\section{Research papers}

Halabi, E. A. et al. Photoregulated fluxional

fluorophores for live-cell super-resolution microscopy with no apparent photobleaching. Nat. Commun. 10, 1232 (2019). 